Emmanuel João Nogueira Leal da Silva ${ }^{1,2}$ Wagner Pereira Coutinho Filho ${ }^{3}$ Aurimar de Oliveira Andrade ${ }^{4}$ Daniel Rodrigo Herrera Morante ${ }^{1}$ Raphael Hirata Junior ${ }^{5}$

Tauby de Souza Coutinho Filho ${ }^{6}$ Renato Liess Krebs ${ }^{6}$

'Doctorando en Clínica Odontológica, área de Endodoncia, Facultad de Odontología de Piracicaba Universidad Estadual de Campinas (FOP-UNICAMP), SP-Brasil.

${ }^{2}$ Profesor Colaborador del Programa de Posgrado en Endodoncia de la Universidad del Estado de Rio de Janeiro (UERJ), RJ-Brasil.

${ }^{3}$ Profesor de la Escuela de Odontología del Centro Universitario Serra dos Órgãos (UNIFESO), RJ-Brasil.

${ }^{4}$ Maestria en Odontología por la Universidad Federal Fluminense (UFF), RJ-Brasil.

Federal Fluminense (UFF), RJ-Brasil. Inmunología de la Universidad del Estado de Rio de Janeiro (UERJ), RJ-Brasil.

${ }^{6}$ Profesor del Departamento de Odontología Restauradora, área de Endodoncia, Universidad del Estado de Rio de Janeiro (UERJ), RJ-Brasil.

\section{Correspondencia}

Daniel Rodrigo Herrera Morante Av. Limeira 901, Areão, Piracicaba-São Paulo, Brasil

e-mail: dani_hm76@ hotmail.com

Recibido : 30 de junio de 2011

Aceptado : 15 de noviembre de 2011

\section{Introducción}

Gran parte de las patologías de origen endodóntico son causadas por la presencia de microorganismos en el sistema de conductos radiculares (SCR). En la mayoría de los casos, los agentes etiológicos son las bacterias, pudiendo estar presentes también levaduras y hongos. Así, el éxito en el tratamiento de la patología endodóntica depende del control de la infección microbiana en el SCR (1).

La infección endodóntica no es plausible de remisión espontánea por la acción de los mecanismos de defensa del organismo, así como tampoco puede ser combatida por antibióticoterapia sistémica. La pulpa dental necrosada no tiene vasos sanguíneos que puedan transportar células de defensa ni antibióticos

\title{
Efecto antimicrobiano de la terapia fotodinámica sobre Enterococcus faecalis, estudio in vitro
}

Silva EJNL, Coutinho-Filho WP, Andrade AO, Herrera DR, Hirata-Junior R, Coutinho-Filho TS, Krebs RL. Efecto antimicrobiano de la terapia fotodinámica sobre Enterococcus faecalis, estudio in vitro. Rev Estomatol Herediana. 2011; 21(4):185-189.

\section{RESUMEN}

OBJETIVO: Evaluar el efecto antibacteriano, in vitro, de la Terapia Fotodinámica (PDT) sobre Enterococcus faecalis. MATERIAL Y MÉTODOS: Fueron preparadas suspensiones bacterianas a partir de cepas clínicas de E. faecalis. Estas suspensiones fueron divididas en tres grupos experimentales (1L, $2 \mathrm{~L}$ y $3 \mathrm{~L}$ ) y un grupo control (GC). Utilizando un equipo Twin Laser conteniendo como medio activo un diodo de GaAIAs, los grupos experimentales recibieron respectivamente dosis de energía de $1 \mathrm{~min}$ a $60 \mathrm{~J} / \mathrm{cm} 2,2 \mathrm{~min}$ a $120 \mathrm{~J} / \mathrm{cm} 2$ y $3 \mathrm{~min}$ a $180 \mathrm{~J} / \mathrm{cm} 2$. Las suspensiones fueron diluidas, sembradas e incubadas para posterior contaje de Unidades Formadoras de Colonia por $\mathrm{mL}(\mathrm{UFC} / \mathrm{mL})$. Los valores de UFC fueron analizados estadísticamente por ANOVA y test de Tukey $(p<0,05)$. RESULTADOS: Los grupos $1 \mathrm{~L}, 2 \mathrm{~L}$ y $3 \mathrm{~L}$ mostraron una reducción de $99,88 \%, 99,86 \%$ y $99,92 \%$ respectivamente. Siendo que esta reducción de UFC/mL fue estadísticamente significativa $(\mathrm{p}<0,05)$. Sin embargo, no fueron encontradas diferencias significativas entre los tres grupos experimentales, sometidos a diferentes tiempos de PDT $(\mathrm{p}>0,05)$. CONCLUSIÓN: La PDT es una alternativa eficaz para la eliminación de E. faecalis, microorganismo frecuentemente asociado a casos de fracaso endodóntico.

Palabras clave: Enterococus faecalis / FOTOQUIMIOTERAPIA / INFECCIÓN ENDODÓNTICA.

Antimicrobial effect of photodynamic therapy against Enterococcus faecalis, in vitro ABSTRACT

OBJECTIVE: To evaluate in vitro, the antibacterial effect of photodynamic therapy (PDT) against Enterococcus faecalis. MATERIAL AND METHODS: Bacterial suspensions were prepared from clinical strains of $E$. faecalis. These suspensions were divided into three experimental groups (1L, $2 \mathrm{~L}$ and $3 \mathrm{~L}$ ) and a control group (CG). The Twin Laser equipment with a GaAIAs diode was used to expose experimental groups to PDT as follow: $1 \mathrm{~min}$ at $60 \mathrm{~J} / \mathrm{cm} 2,2 \mathrm{~min}$ at $120 \mathrm{~J} / \mathrm{cm} 2$ and $3 \mathrm{~min}$ at 180 $\mathrm{J} / \mathrm{cm} 2$. After the experimental treatments, $0.1 \mathrm{~mL}$ aliquots of the suspensions were diluted, seeded and incubated for further counting of Colony Forming Units per $\mathrm{mL}(\mathrm{CFU} / \mathrm{mL})$. The results were analyzed by analysis of variance and Tukey's test $(\mathrm{p}<0.05)$. RESULTS: The groups $1 \mathrm{~L}, 2 \mathrm{~L}$ and $3 \mathrm{~L}$ showed a reduction of $99.88 \%, 99.86 \%$ and $99.92 \%$ respectively $(\mathrm{p}<0.05)$. However, no significant differences were found between experimental times of PDT $(p>0.05)$. CONCLUSION: The results showed that PDT was effective in reducing the number of viable cells of $E$. faecalis, a microorganism frequently associated with failure of root canal failure.

Key words: Enterococcus faecalis / PHOTOCHEMOTHERAPY / ENDODONTIC INFECTION.

para la zona. De esta manera, y debido a la localización anatómica de la infección endodóntica, existe la necesidad de intervención profesional (2).

El proceso de desinfección se inicia con la preparación químicomecánica (PQM) del SCR, por medio de la acción concomitante de la instrumentación mecánica y de las sustancias químicas auxiliares (SQA). Sin embargo, esta etapa no elimina por completo los microorganismos presentes en procesos infecciosos crónicos, donde los microorganismos de la luz del conducto se propagan por todo el SCR, lo que incluye túbulos dentinarios, conductos accesorios, ramificaciones laterales, colaterales y delta apical $(3,4)$. La misma situación puede presentarse frente a conductos curvos, estrechos o con accidentes anatómicos internos que dificulten el acceso de los instrumentos y las SQA (5). La imposibilidad de erradicar todos los microorganismos durante el tratamiento endodóntico, sumado a los factores anatómicos, permiten la colonización del ápice radicular y de los tejidos periapicales, perpetuando la infección (6).

La microbiota presente en conductos radiculares obturados con fracaso en el tratamiento endodóntico difiere tanto cuantitativa como cualitativamente de aque1la normalmente encontrada en dientes necrosados y no tratados. Las infecciones secundarias y persistentes presentan un número limitado de microorganismos con predominio de anaerobios facultativos Gram-positivos. Por otro lado, 
una infección polimicrobiana con predominio de anaerobios estrictos Gram-negativos está comúnmente asociada a conductos no tratados endodónticamente $(7,8)$.

Dentro de los microorganismos resistentes a la terapia endodóntica, Enterococcus faecalis, coco entérico Gram-positivos, anaerobio facultativo, es altamente relacionado a casos de fracaso endodóntico (9-14). E. faecalis puede sobrevivir a la instrumentación, irrigación y medicación intraconducto (MIC), sobreviviendo en túbulos dentinarios, consiguiendo reinfectar el canal obturado (9).

La utilización del láser en odontología ha evolucionado en los últimos años, lo que incluye el láser de Érbio (Er:YAG) con puntas específicas para utilización en contacto, tanto en Endodoncia como en Periodoncia. El uso de un láser de baja potencia, tanto como laserterapia (que estimula la biomodulación, sus efectos analgésicos y antiinflamatorios) como en la terapia fotodinámica (que asocia una fuente luminosa a un colorante específico para la reducción microbiana) han ganado cada vez más espacio dentro de las especialidades odontológicas (15).

La terapia fotodinámica (PDT) inicialmente preconizada para el tratamiento del cáncer (16), ha venido siendo investigada también para el tratamiento de enfermedades infecciosas. Debido a que la gran mayoría de microorganismos orales no absorbe la luz visible de los láser de baja potencia, es necesaria la utilización de un colorante que se fije a ellos, atrayendo la luz del láser. Cuando el fotosintetizador es irradiado con una luz de longitud de onda adecuada, la molécula es excitada produciendo una serie de transferencias de energía molecular. Todo esto lleva a la liberación de radicales libres y/o oxígeno singlete, una especie altamente reactiva y citotóxica, lo que resulta en muerte celular (17).

El verde de malaquita (VM) es un colorante utilizado de rutina en clínica odontológica, con capacidad de interactuar con varias sustancias orgánicas, principalmente con el biofilm oral adherido a los elementos dentales. El VM presenta una banda de absorción en la región roja del espectro electromagnético (18), siendo de fácil tránsito por la membrana celular tanto de bacterias Gram-positivas como de Gramnegativas. Este colorante catiónico pertenece a la familia de los trifenilmetanos pudiendo ser utilizado como un potente fotosintetizador, ya que promueve la disipación del potencial de la membrana celular (19). Así, el objetivo de este estudio fue evaluar el efecto antibacteriano, in vitro, de la PDT sobre E. Faecalis.

\section{Material y métodos}

Cepas clínicas fueron colectadas y aisladas de dientes con periodontitis apical crónica, obteniendo entre ellas cepas de E. faecalis, lo que fue confirmado por la prueba bioquímica API Strep (BioMérieux SA, Marcy-l'Etoile, Francia), las cuales fueron congeladas $(-20 \mathrm{oC})$ en solución de Skim Milk al 10\% (Difco Laboratories, Sparks, USA). Antes de los ensayos experimentales, las cepas fueron descongeladas y sembradas en placas Petri conteniendo Agar Triptone Soya (TSA, Difco Laboratories, Sparks, EUA).

Utilizando Caldo Triptone Soya (TSB, Difco Laboratories, Sparks, EUA) fueron preparadas e incubadas $\left(37^{\circ} \mathrm{C} / 24 \mathrm{hrs}\right)$ suspensiones de E. faecalis. Después de confirmado el crecimiento bacteriano, las suspensiones fueron centrifugadas (14000 rpm/2min) y el sobrenadante lavado tres veces en una solución tampón (PBS, Sodium Phosphate Buffer, Difco Laboratories, Sparks, EUA). Fueron colectadas bacterias y llevadas a solución salina estéril para la preparación de suspensiones estandarizadas en 0,5 de la escala

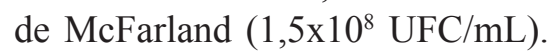
Cada tubo $(n=24)$ conteniendo 900 $\mu \mathrm{L}$ de suspensión fue irradiado desde la base. El equipo utilizado fue el Twin Laser (MM Optics, São Paulo, Brasil) conteniendo como medio activo un diodo de GaAIAs, emitiendo en $660 \mathrm{~nm}$ con $40 \mathrm{~mW}$ de potencia.

Los tubos fueron distribuidos en tres grupos experimentales y un grupo control. El grupo control (GC) no recibió VM ni láser; los grupos experimentales $(1 \mathrm{~L}),(2 \mathrm{~L})$ y $(3 \mathrm{~L})$ recibieron $0,01 \% \mathrm{~m} / \mathrm{v}$ de VM y después de un minuto fueron expuestos al láser por periodos de $1 \mathrm{~min}$ con dosis de energía de $60 \mathrm{~J} /$ $\mathrm{cm}^{2}, 2 \mathrm{~min}$ a $120 \mathrm{~J} / \mathrm{cm}^{2}$ y $3 \mathrm{~min}$ a $180 \mathrm{~J} / \mathrm{cm}^{2}$, respectivamente. Fueron adicionados $100 \mu \mathrm{L} 0,1 \% \mathrm{VM}$ a los grupos experimentales, resultando en una concentración final de $0,01 \%$ $\mathrm{m} / \mathrm{v}$.

Las suspensiones fueron diluidas $\left(10^{-3}\right)$, y alícuotas de $10 \mu \mathrm{L}$ de cada grupo fueron llevadas a placas conteniendo TSA e incubadas $\left(48 \mathrm{hr} / 37^{\circ} \mathrm{C}\right)$ en estufa de $5 \%$ de $\mathrm{CO}_{2}$ para posterior conteo de Unidades Formadoras de Colonia por $\mathrm{mL}$ (UFC/mL). Los valores de UFC fueron analizados estadísticamente por ANOVA y test de Tukey $(\mathrm{p}<0,05)$.

\section{Resultados}

Los grupos $1 \mathrm{~L}, 2 \mathrm{~L}$ y $3 \mathrm{~L}$ mostraron una reducción de 99,88\%, $99,86 \%$ y $99,92 \%$ respectivamente. Siendo que esta reducción de UFC/ $\mathrm{mL}$ fue estadísticamente significativa $(p<0,05)$. Sin embargo, no fueron encontradas diferencias significativas entre los tres grupos 
Tabla 1. Media de UFC/mL inicial y final de E. faecalis en los diferentes grupos experimentales así como la desviación estándar y su significancia estadística.

\begin{tabular}{lll}
\hline & UFC inicial & UFC final \\
\hline GC & $1,35 \times 108 \pm 0,00 \mathrm{Aa}$ & $2,91 \times 108 \pm 1,01 \times 108 \mathrm{Bb}$ \\
$1 \mathrm{~L}$ & $1,35 \times 108 \pm 0,00 \mathrm{Ac}$ & $1,66 \times 105 \pm 0,22 \times 105 \mathrm{Bd}$ \\
$2 \mathrm{~L}$ & $1,35 \times 108 \pm 0,00 \mathrm{Ae}$ & $1,83 \times 105 \pm 0,27 \times 105 \mathrm{Bf}$ \\
$3 \mathrm{~L}$ & $1,35 \times 108 \pm 0,00 \mathrm{Ag}$ & $1,12 \times 105 \pm 0,14 \times 105 \mathrm{Bh}$ \\
\hline
\end{tabular}

Para cada columna, medias seguidas de letras mayúsculas iguales no son significativamente diferentes.

Para cada línea, medias seguidas de letras minúsculas iguales no son significativamente diferentes. GC- grupo control (sin PDT); 1L, 2L y 3L- grupos que recibieron 1, 2 y 3 min de PDT respectivamente.

experimentales, sometidos a diferentes tiempos de PDT ( $\mathrm{p}>0,05)$ (Tabla 1).

\section{Discusión}

La eliminación de microorganismos del sistema de conductos radiculares (SCR) es parte importante de los procedimientos de la terapia endodóntica con el fin de obtener el éxito en el tratamiento. La elección de E. Faecalis para este estudio, fue basada en las evidencias científicas que muestran a este microorganismo como el más prevalente en los casos de fracaso del tratamiento endodóntico $(12,13,20)$. La diferencia en la prevalencia de este microorganismo entre las infecciones endodónticas primarias y los casos de fracaso del tratamiento endodóntico se debe lal desequilibrio del ecosistema existente dentro del SCR provocado por una instrumentación y limpieza poco eficientes que propicia la sobrevivencia de microorganismos resistentes $(7,10)$.

La PDT es un eficiente método para la reducción microbiana, habiéndose mostrado eficaz en infecciones localizadas, de poca profundidad y de microbiota conocida; esto le ha valido para ser indicada como una alternativa para la desinfección del SCR (21-27), posibilitando la desinfección en solo una cita. Existen diversos equipos de láser en el mercado, los cuales cubren por completo el espectro visible e infrarrojo. Estos equipos pueden actuar con diversos agentes fotosintetizadores. El láser de GaAlAs utilizado en este estudio, es un láser de baja potencia que emite luz roja con longitud de onda de $660 \mathrm{~nm}$. En este estudio el láser fue regulado en $40 \mathrm{~mW}$ de potencia, de acuerdo con estudios previos donde también fueron obtenidos resultados semejantes (28).

La elección del VM se debe al hecho de que este colorante presenta una banda de absorción en la región roja del espectro electromagnético (18), y por ser de fácil tránsito por la membrana celular tanto de bacterias Gram-positivas como de Gram-negativas. Cabe resaltar la importancia de la elección conjunta del equipo láser y del colorante a ser utilizados en la PDT. El efecto antimicrobiano de la PDT dependerá también de la concentración del colorante. La concentración utilizada en este estudio fue de $0,01 \% \mathrm{w} / \mathrm{v}$ mostrándose efectiva.

Los factores que pueden influenciar en la efectividad de la PDT son (29):

- Diferencias en la concentración y el tipo de colorante.

- Tiempo de irradiación.

- Concentración y estado fisiológico de los microorganismos.
- Tiempo de exposición al láser y densidad de energía.

Nuestros resultados concuerdan con los obtenidos en un estudio previo (30), donde se obtuvo una reducción $99,2 \%$ de E. faecalis utilizando un agente fotosintetizante azul asociado a láser de $685 \mathrm{~nm}$. Otra propiedad importante da PDT es que no induce a resistencia microbiana, siendo plausible de una segunda aplicación (29).

Los resultados encontrados en este trabajo son promisorios cuanto a la aplicación clínica de la PDT. Sin embargo, son necesarios estudios que viabilicen la implementación de un protocolo clínico para casos de infección endodóntica donde la PDT pueda ser utilizada como una alternativa efectiva. Otro factor que debe ser analizado es la posibilidad de que los colorantes utilizados como fotosintetizadores puedan pigmentar el diente a ser tratado con PDT.

\section{Conclusión}

La PDT es una alternativa eficaz para la eliminación de E. faecalis, microorganismo persistente en los casos de fracaso del tratamiento endodóntico.

\section{Referencias bibliográficas}

1. Siqueira JF Jr. Endodontic infections: concepts, paradigms, and perspectives. Oral Surg Oral Med Oral Pathol Oral Radiol Endod. 2002; 94(3):281-93.

2. Siqueira JF Jr, Rôças IN. Polymerase chain reaction-based analysis of microorganisms associated with failed endodontic treatment. Oral Surg Oral Med Oral Pathol Oral Radiol Endod. 2004; 97(1):85-94.

3. Leonardo MR, Almeida WA, Ito IY, da Silva LA. Radiographic and microbiologic evaluation 
of posttreatment apical and periapical repair of root canals of dogs' teeth with experimentally induced chronic lesion. Oral Surg Oral Med Oral Pathol. 1994; 78(2):232-8.

4. Estrela C, Pimenta FC, Ito IY, Bammann LL. Antimicrobial evaluation of calcium hydroxide in infected dentinal tubules. J Endod. 1999; 25(6):416-8.

5. Marchesan MA, Arruda MP, Silva-Sousa YT, Saquy PC, Pecora JD, Sousa-Neto MD. Morphometrical analysis of cleaning capacity using nickel-titanium rotary instrumentation associated with irrigating solutions in mesio-distal flattened root canals. J Appl Oral Sci. 2003; 11(1):55-9.

6. Abou-Rass M, Bogen G. Microorganisms in closed periapical lesions. Int Endod J. 1998; 31(1):39-47.

7. Sundqvist G, Figdor D, Persson $\mathrm{S}$, Sjögren U. Microbiologic analysis of teeth with failed endodontic treatment and the outcome of conservative retreatment. Oral Surg Oral Med Oral Pathol Oral Radiol Endod. 1998; 85(1):86-93.

8. Molander A, Reit C, Dahlén G, Kvist T. Microbiological status of root-filled teeth with apical periodontitis. Int Endod J. 1998; 31(1):1-7.

9. Love RM. Enterococcus faecalis--a mechanism for its role in endodontic failure. Int Endod J. 2001; 34(5):399-405.

10. Siqueira JF Jr, Rôças IN, Souto $\mathrm{R}$, de Uzeda M, Colombo AP. Actinomyces species, streptococci, and Enterococcus faecalis in primary root canal infections. J Endod. 2002; 28(3):168-72.

11.Evans MD, Baumgartner JC, Khemaleelakul SU, Xia T. Effi- cacy of calcium hydroxide: chlorhexidine paste as an intracanal medication in bovine dentin. J Endod. 2003; 29(5):338-9.

12. Gomes BP, Pinheiro ET, GadêNeto CR, Sousa EL, Ferraz CC, Zaia AA, Teixeira FB, SouzaFilho FJ. Microbiological examination of infected dental root canals. Oral Microbiol Immunol. 2004; 19(2):71-6.

13. Gomes BP, Pinheiro ET, Sousa EL, Jacinto RC, Zaia AA, Ferraz CC, de Souza-Filho FJ. Enterococcus faecalis in dental root canals detected by culture and by polymerase chain reaction analysis. Oral Surg Oral Med Oral Pathol Oral Radiol Endod. 2006; 102(2):247-53.

14. Stuart CH, Schwartz SA, Beeson TJ, Owatz CB. Enterococcus faecalis: its role in root canal treatment failure and current concepts in retreatment. J Endod. 2006; 32(2):93-8.

15.Krebs RL. Análise da viabilidade celular de um monobiofilme de Streptococcus mutans submetido a terapia fotodinâmica [Tese Doutorado em Odontologia]. [Rio de Janeiro]: Faculdade de Odontologia da Universidade do Estado do Rio de Janeiro; 2007.

16. Ackroyd R, Kelty C, Brown N, Reed M. The history of photodetection and photodynamic therapy. Photochem Photobiol. 2001; 74(5):656-69.

17.Zanin ICJ, Brugnera-Jr A, Zani F, Gonçalves RB. Terapia fotodinâmica na odontología (TFD). RGO. 2003; 51(3):179-82.

18. Yamaoka K, Sasai R. Pulsed Electric Linear Dichroism of Triphenylmethane Dyes Adsorbed on Montmorillonite K10 in Aqueous Media. J Colloid Interface Sci. 2000; 225(1):82-93.
19.Kowaltowski AJ, Turin J, Indig GL, Vercesi AE. Mitochondrial effects of triarylmethane dyes. J Bioenerg Biomembr. 1999; 31(6):581-90.

20.Zoletti GO, Siqueira JF Jr, Santos KR. Identification of Enterococcus faecalis in root-filled teeth with or without periradicular lesions by culture-dependent and-independent approaches. J Endod. 2006; 32(8):722-6.

21.Garcez AS, Ribeiro MS, Tegos GP, Núñez SC, Jorge AO, Hamblin MR. Antimicrobial photodynamic therapy combined with conventional endodontic treatment to eliminate root canal biofilm infection. Lasers Surg Med. 2007; 39(1):59-66.

22.Soukos NS, Chen PS, Morris JT, Ruggiero K, Abernethy AD, Som S, Foschi F, Doucette S, Bammann LL, Fontana CR, Doukas AG, Stashenko PP. Photodynamic therapy for endodontic disinfection. J Endod. 2006; 32(10):979-84.

23.Bergmans L, Moisiadis $\mathrm{P}, \mathrm{Hu}-$ ybrechts B, Van Meerbeek B, Quirynen M, Lambrechts P. Effect of photo-activated disinfection on endodontic pathogens ex vivo. Int Endod J. 2008; 41(3):227-39.

24.Ng R, Singh F, Papamanou DA, Song X, Patel C, Holewa C, Patel N, Klepac-Ceraj V, Fontana CR, Kent R, Pagonis TC, Stashenko PP, Soukos NS. Endodontic photodynamic therapy ex vivo. J Endod. 2011; 37(2):217-22.

25. Garcez AS, Nuñez SC, Hamblim MR, Suzuki H, Ribeiro MS. Photodynamic therapy associated with conventional endodontic treatment in patients with antibiotic-resistant microflora: a preliminary report. J Endod. 2010; 36(9):1463-6. 
26. Souza LC, Brito PR, de Oliveira JC, Alves FR, Moreira EJ, Sampaio-Filho HR, Rôças IN, Siqueira JF Jr. Photodynamic therapy with two different photosensitizers as a supplement to instrumentation/irrigation procedures in promoting intracanal reduction of Enterococcus faecalis. J Endod. 2010; 36(2):292-6.

27.Fonseca MB, Júnior PO, Pallota RC, Filho HF, Denardin OV, Rapoport A, Dedivitis RA, Vero- nezi JF, Genovese WJ, Ricardo AL. Photodynamic therapy for root canals infected with Enterococcus faecalis. Photomed Laser Surg. 2008; 26(3):209-13.

28. Matevski D, Weersink R, Tenenbaum HC, Wilson B, Ellen RP, Lépine G. Lethal photosensitization of periodontal pathogens by a red-filtered Xenon lamp in vitro. J Periodontal Res. 2003; 38(4):428-35.

29. Wilson M, Mia N. Sensitisation of Candida albicans to killing by low-power laser light. J Oral Pathol Med. 1993; 22(8):354-7. 30. Silva Garcez A, Núñez SC, LageMarques JL, Jorge AO, Ribeiro MS. Efficiency of $\mathrm{NaOCl}$ and laser-assisted photosensitization on the reduction of Enterococcus faecalis in vitro. Oral Surg Oral Med Oral Pathol Oral Radiol Endod. 2006; 102(4):e93-8. 